\title{
A novel variant of FGFR3 causes proportionate short stature
}

\author{
Sarina G Kant ${ }^{1}$, Iveta Cervenkova ${ }^{2}$, Lukas Balek ${ }^{2}$, Lukas Trantirek ${ }^{3}$, Gijs W E Santen ${ }^{1}$, \\ Martine C de Vries ${ }^{4}$, Hermine A van Duyvenvoorde', Michiel J R van der Wielen ${ }^{1}$, \\ Annemieke J M H Verkerk ${ }^{5}$, André G Uitterlinden ${ }^{5}$, Sabine E Hannema ${ }^{4}$, Jan M Wit ${ }^{4}$, \\ Wilma Oostdijk ${ }^{4}$, Pavel Krejci ${ }^{2,6, *}$ and Monique Losekoot ${ }^{1, *}$
}

Correspondence should be addressed to S G Kant Email s.g.kant@lumc.nl

\begin{abstract}
Objective: Mutations of the fibroblast growth factor receptor 3 (FGFR3) cause various forms of short stature, of which the least severe phenotype is hypochondroplasia, mainly characterized by disproportionate short stature. Testing for an FGFR3 mutation is currently not part of routine diagnostic testing in children with short stature without disproportion.

Design: A three-generation family A with dominantly transmitted proportionate short stature was studied by whole-exome sequencing to identify the causal gene mutation. Functional studies and protein modeling studies were performed to confirm the pathogenicity of the mutation found in FGFR3. We performed Sanger sequencing in a second family B with dominant proportionate short stature and identified a rare variant in FGFR3.

Methods: Exome sequencing and/or Sanger sequencing was performed, followed by functional studies using transfection of the mutant FGFR3 into cultured cells; homology modeling was used to construct a three-dimensional model of the two FGFR3 variants.

Results: A novel p.M528I mutation in FGFR3 was detected in family A, which segregates with short stature and proved to be activating in vitro. In family B, a rare variant (p.F384L) was found in FGFR3, which did not segregate with short stature and showed normal functionality in vitro compared with WT.

Conclusions: Proportionate short stature can be caused by a mutation in FGFR3. Sequencing of this gene can be considered in patients with short stature, especially when there is an autosomal dominant pattern of inheritance. However, functional studies and segregation studies should be performed before concluding that a variant is pathogenic.
\end{abstract}

\section{Introduction}

One of the most frequently mutated genes in patients with short stature is the fibroblast growth factor receptor 3 (FGFR3) gene. Mutations in FGFR3 have been identified in several skeletal disorders (1), and almost all reported FGFR3 mutations to date cause constitutive activation of the receptor, resulting in impaired endochondral bone growth (2).
(C) 2015 European Society of Endocrinology Printed in Great Britain
Hypochondroplasia (HCH; OMIM 146000) is the least severe form of skeletal dysplasia caused by an FGFR3 mutation. Although the expression of clinical features is variable, $\mathrm{HCH}$ is mostly characterized by short stature with disproportionately short limbs, relative macrocephaly, a normal face, and short and broad hands and feet. The most important radiologic features are shortening of long

Published by Bioscientifica Ltd. 
bones, narrowing (or failure to widen) of the lumbar interpedicular distances, short femoral neck, and square ilia (3).

We studied two families in which several members had short stature, normal body proportions, and no evident radiologic skeletal abnormalities, transmitted in an autosomal-dominant fashion. In the first family, a novel missense mutation in FGFR3 was revealed by wholeexome sequencing, which cosegregated with short stature and showed increased FGFR3, STAT, and ERK/MAP kinase activation in vitro. In the second family, Sanger sequencing revealed a rare variant in FGFR3, which did not fully cosegregate with short stature and did not activate ERK/MAP kinase.

\section{Subjects and methods}

\section{Patients}

Family A $\triangleright$ The index patient (Fig. 1; parents of the patient gave written permission for publication of these photographs) was a female of Dutch ancestry, born after an uneventful pregnancy at a gestational age of 41 weeks. Birth weight was $3025 \mathrm{~g}$ (-1.3 SDS) (4). Birth length was not measured. In the first years, her growth velocity decreased, but from 4 years onwards height remained stable at -3 SDS. No further health problems were noted.
Psychomotor development was normal. Her most recent examination has been at age 10.5 years, and shown a height $(\mathrm{H})$ of $125.1 \mathrm{~cm}$ (-3.3 SDS) (5), weight $30 \mathrm{~kg}$ (BMI 19.2; 1.1 SDS) (6), head circumference (HC) $52.2 \mathrm{~cm}(-0.5$ SDS) (7), span $129 \mathrm{~cm}$, sitting height (SH) $66.8 \mathrm{~cm}(\mathrm{SH}: \mathrm{H}$ ratio $0.534,=1.0 \mathrm{SDS}$ ) (8). No physical anomalies were noted. A skeletal survey showed no abnormalities (Fig. 1). At a calendar age of 10.02 years, bone age was 9.89 years. Serum IGFI and IGFBP3 levels were within normal ranges. Karyotyping and SHOX mutation analysis showed normal results. SNP array (262K NspI Affymetrix array) revealed a $155.5 \mathrm{~kb}$ deletion of chromosome 9q31.3 (110.595.815110.751.335; hg18), containing four genes, which did not segregate with the short stature in the family.

The pedigree is shown in Fig. 2. The mother also had short stature: H $151.4 \mathrm{~cm}$ (-3.1 SDS), HC $55.8 \mathrm{~cm}(+0.3$ SDS), span $153.8 \mathrm{~cm}, \mathrm{SH} 81.9 \mathrm{~cm}$ (SH:H ratio 0.541:1.1 SDS). Height of the maternal grandmother was 148.1 (-3.6 SDS), HC $54.2 \mathrm{~cm}$ (-0.7 SDS), span $150.7 \mathrm{~cm}, \mathrm{SH}$ $80.4 \mathrm{~cm}$ (SH:H ratio $0.541,=1.1 \mathrm{SDS})$. The father had a normal stature and so did the brother of the index patient and the sister of the maternal grandmother.

Family B $\triangleright$ The index patient was a male of Dutch ancestry born after an uneventful pregnancy at a gestational age of 42 weeks. Birth weight was $3641 \mathrm{~g}$ ( -0.3 SDS $)$. Birth length was not measured. After 6
A

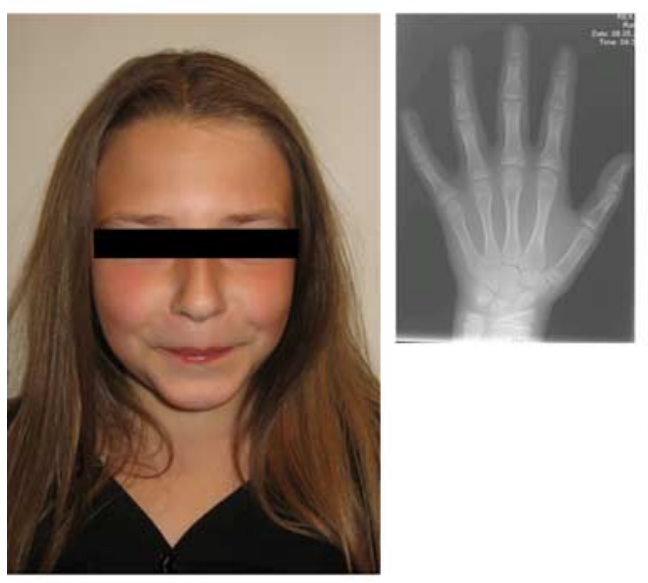

C

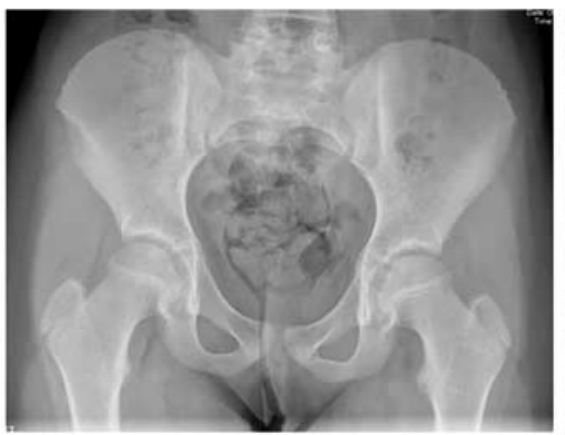

D

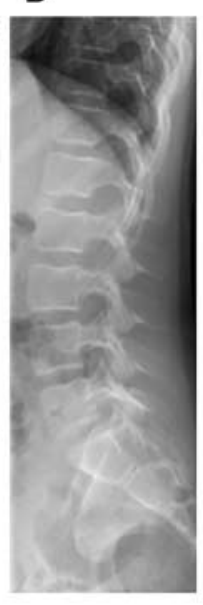

E

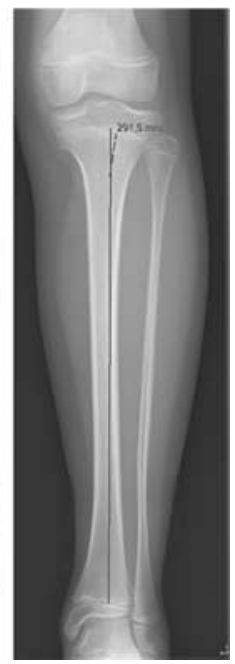

\section{Figure 1}

Clinical photograph (A) and X-rays (B, C, D and E) of the index patient at the age of 10.6 years. No evident radiologic abnormalities were noted (parents of the patient gave permission for publication of these photographs). A full colour version of this figure is available at http://dx.doi.org/10.1530/ EJE-14-0945. 


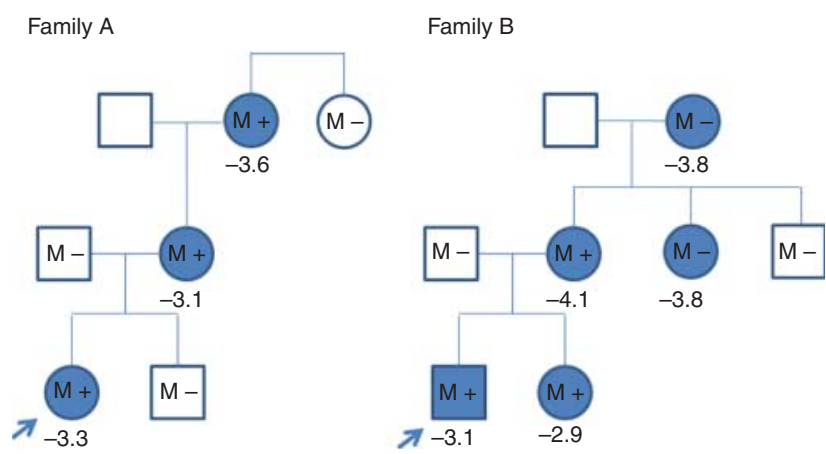

\section{Figure 2}

Pedigrees of families A and B. Filled symbols represent family members with short stature. Underneath these symbols height SDS is displayed. $\mathrm{M}+$, familial variant in FGFR3 is present; $\mathrm{M}-$, familial variant in FGFR3 is not present. In both maternal grandfathers sequence analysis of FGFR3 has not been performed. A full colour version of this figure is available at http://dx.doi.org/10.1530/EJE-14-0945.

months, his growth velocity decreased and from 4 years onward height remained stable at -3.2 SDS. No further health problems were noted. Psychomotor development was normal. His most recent examination has been at age 5.3 years, and shown $\mathrm{H} 99.7 \mathrm{~cm}(-3.1 \mathrm{SDS})$, weight $16.5 \mathrm{~kg}$ (BMI 16.3; 0.8 SDS), HC $50.5 \mathrm{~cm}$ (-0.6 SDS), and $\mathrm{SH} 57.0 \mathrm{~cm}$ (SH:H ratio 0.57, =1.7 SDS). No physical anomalies were noted. A skeletal survey showed no abnormalities. Bone age was 4.85 years at a calendar age of 4.23 years (within normal limits). Serum insulin-like growth factor 1 (IGF1) and IGFBP3 levels were within normal ranges. Whole-genome SNP array and mutation analysis for NPR2 and SHOX showed normal results.

As shown in the pedigree (Fig. 2), the mother also had proportionate short stature: $\mathrm{H} 144.6 \mathrm{~cm}(-4.1$ SDS), HC +1.0 SDS, span $149 \mathrm{~cm}$, and SH:H ratio 0.54:1.7 SDS). The same holds for the 3-year-old sister (H 83.8 (-2.9 SDS), HC 49.5 (0.3 SDS), span $81.3 \mathrm{~cm}$, and $\mathrm{SH}: \mathrm{H}$ ratio 0.59 (0.8 SDS)). The sister of the mother and the maternal grandmother are both $146 \mathrm{~cm}$ ( $-3.8 \mathrm{SDS})$. The father was actually rather tall, $191.1 \mathrm{~cm}$ (1.0 SDS). The brother of the mother is $175 \mathrm{~cm}(-1.3 \mathrm{SDS})$.

\section{DNA sequencing}

The exomes of the index patient, her parents, brother, maternal grandmother, and the grandmother's sister in family A were sequenced and the variants were filtered based on cosegregation, predicted impact on protein function, conservation and presence in public databases. The exomes were captured by the NimbleGen SeqCap EZ V2 kit followed by Illumina-paired end sequencing $(2 \times$ $100 \mathrm{bp}$ ) with at least $40 \times$ mean coverage. Downstream analysis was performed with an in-house pipeline (9). The variants discovered by whole exome sequencing were confirmed by Sanger sequencing. Sequencing of the coding region of FGFR3 was performed according to standard procedures. Investigations were approved by the local ethical committee.

\section{Western blotting, vectors and transfection}

Rat chondrosarcoma (RCS) chondrocytes and 293T and NIH3T3 cells were propagated in DMEM medium supplemented with 10\% FBS and antibiotics (Invitrogen). The cells were lysed in Laemmli buffer. Protein samples were resolved by SDS-PAGE, transferred onto a PVDF membrane, and visualized by chemiluminiscence (Thermo Scientific, Rockford, IL, USA). Western blotting (WB) signal was quantified by Image J Software (National Institutes of Health, USA). The following antibodies were used: FGFR3, actin (Santa Cruz Biotechnology), ERK, pERK, pFGFR, pSTAT1, STAT1, pSTAT3, STAT3 (Cell Signaling, Beverly, MA, USA). The cells were transiently transfected using FuGENE6, according to the manufacturer's protocol (Roche). Vectors expressing V5-tagged human FGFR3 were made by cloning full-length human FGFR3 into the pcDNA3.1 backbone (Invitrogen); mutants were generated via site-directed mutagenesis (Agilent Technologies, Santa Clara, CA, USA).

\section{Protein modeling}

Homology modeling was employed to construct a threedimensional structural model of the asymmetric dimer of the cytosolic tyrosine kinase domain (TKD) of the FGFR3. The crystal structure of the kinase domain dimer of the FGFR3 analog, namely epidermal growth factor receptor (EGFR) (PDB ID: 2GS6), was used as a template. The programs PHYRE (10) and UCSF Chimera (11) were used for generation of the dimer model.

\section{Results}

Whole-exome sequencing of all affected family members of family A revealed a novel mutation (NM_000142.4: c.1584G > T; Chr4(GRCh37): g.1807335G > T; p.Met 528Ile) in FGFR3. Detailed sequencing characteristics of 


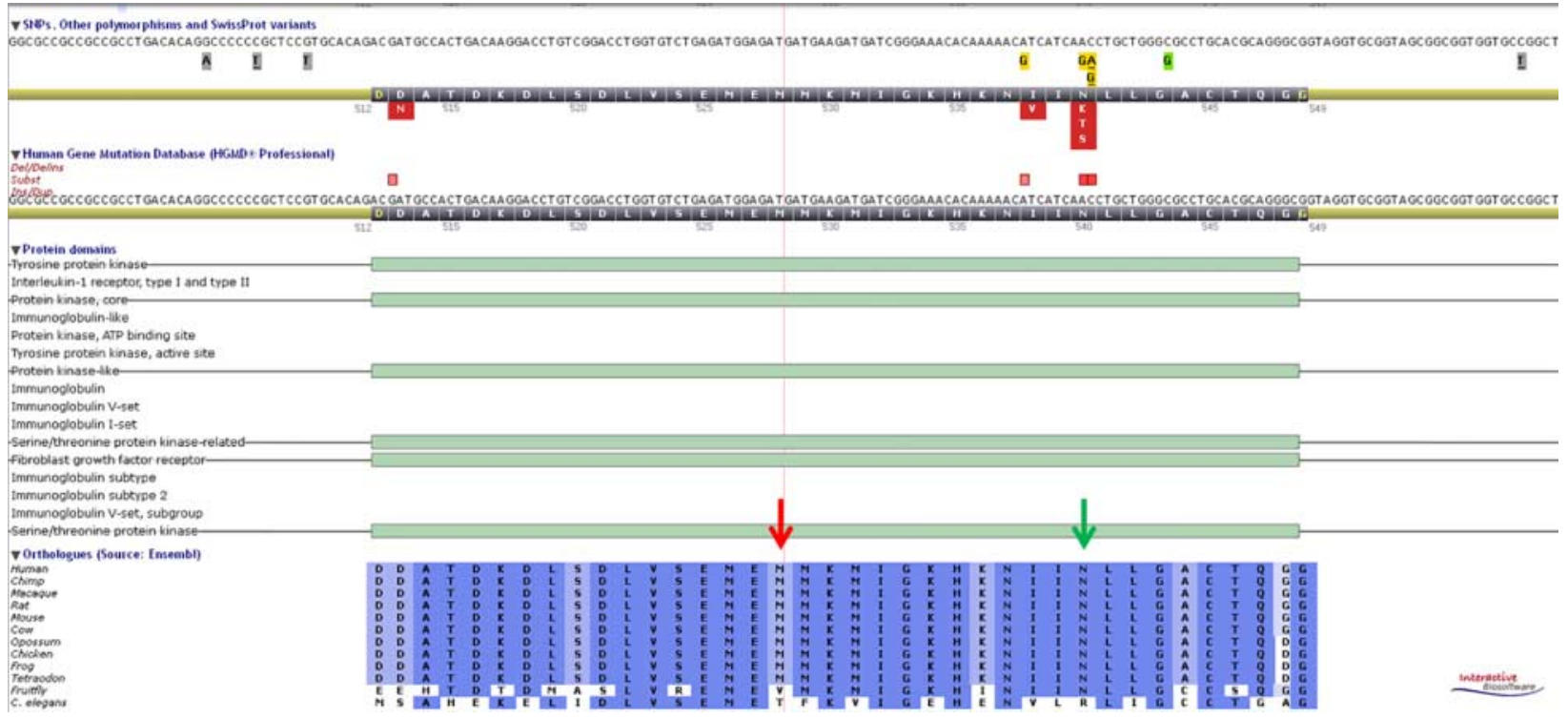

\section{Figure 3}

Schematic representation of location of the p.M528I mutation in the tyrosine protein kinase domain. A full colour version of this figure is available at http://dx.doi.org/10.1530/EJE-14-0945.

all performed exome sequencing analyses are listed in Supplementary Table 1 , see section on supplementary data given at the end of this article. The p.M528I mutation is located in the TKD, close to the known $\mathrm{HCH}$ mutation p.N540K (Fig. 3). The variant was predicted to be possibly damaging in several in silico prediction programs (align GVGD: C0 (GV: 81.04 - GD: 10.12), SIFT: tolerated (score: 0.29, median: 3.24), MutationTaster: disease causing (P value: 1$)$, PolyPhen: predicted to be probably damaging (HumDiv score 0.995 (sensitivity 0.68, specificity 0.97), and HumVar score 0.978 (sensitivity 0.58, specificity 0.94)). Sanger sequencing confirmed the presence of this mutation in the index, the affected mother and maternal grandmother, and was not present in the normal statured brother, father, and unaffected sister of the maternal grandmother.

Subsequent transfection studies of the mutant FGFR3 into cultured cells proved the mutation to be activating, with the activation of FGFR3, STAT, and ERK/MAP kinase similar to the G380R mutation that causes achondroplasia (ACH) (Fig. 4).

Homology modeling showed a three-dimensional model of the asymmetric dimer of the cytosolic TKD of FGFR3, which highlights the position of Met528 at the dimerization interface essential for activation of the TKD (Fig. 5).

In the index patient of family $\mathrm{B}$, a rare variant (NM_000142.4: c.1150T >C, r4(GRCh37): g.1806131T >C,
p.Phe384Leu) was found in the FGFR3 gene with Sanger sequencing. This variant was also demonstrated in the affected sister and mother, but not in the affected grandmother and maternal aunt. The variant was predicted to be benign in several in silico prediction programs (align GVGD: C0 (GV: 204.97 - GD: 0.00), SIFT: tolerated (score: 1, median: 3.24), MutationTaster: polymorphism ( $P$ value: 0.918), PolyPhen: predicted to be benign (HumDiv score 0.003161-163) (sensitivity 0.98, specificity 0.44 and HumVar score 0.008 sensitivity 0.96 , specificity 0.48)), which has been reported by Trujillo-Tiebas et al. (12) as a variant of unknown clinical significance and by Bodian et al. (13) as detected in a healthy ancestrally diverse cohort. The variant is described in dbSNP as rs17881656 (MAF/MinorAlleleCount: $C=0.002 / 4)$ in populations from different ethnic origin. Transfection studies of the mutant FGFR3 into RCS chondrocytes could not prove the mutation to be activating (data not shown).

\section{Discussion}

The association between a novel heterozygous p.M528I mutation in FGFR3 and proportionate short stature shows that the already quite wide spectrum of clinical presentations of heterozygous FGFR3 mutations should be further extended to proportionate short stature. This implies that for such patients FGFR3 should be considered a candidate gene, particularly if short stature is 


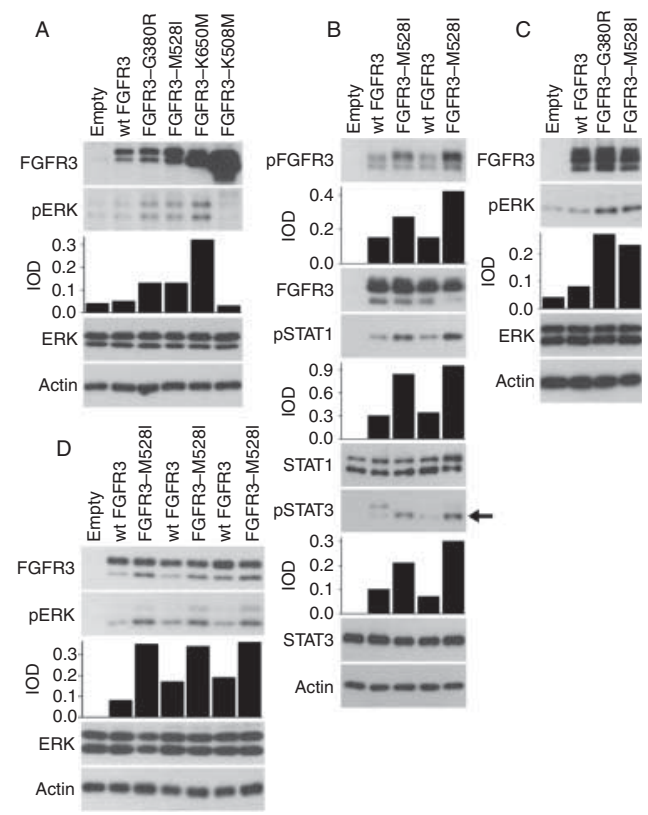

\section{Figure 4}

(A) RCS chondrocytes were transfected with WT FGFR3, FGFR3M528I, known activating mutants (G380R, K650M), kinaseinactive mutant $\mathrm{K} 508 \mathrm{M}$, or empty plasmid. The cells were grown for $48 \mathrm{~h}$ and analyzed by western blotting (WB). The levels of ERK/MAP kinase activation by FGFR3 were determined by WB with antibody recognizing ERK only when phosphorylated ( $p)$ at T202/T204; pERK signal was quantified by densitometry and graphed (integrated optical density, IOD). Total ERK and actin levels serve as loading controls, total FGFR3 levels serve as control for transfection. Note the different levels of FGFR3mediated ERK activation that are the highest in thanatophoric dyplasia mutation K650M. FGFR3-M528I activates ERK to the same extent as achondroplasia-mutant G380R. (B) 293T cells were transfected as indicated and analyzed for activating phosphorylation (p) of FGFR3 ${ }^{\text {Y653/Y654 }}$, STAT1 $^{\text {Y701 }}$, and STAT3 ${ }^{\text {Y703 }}$ by WB. Signal was quantified by densitometry and graphed (IOD). Total FGFR3, STAT1, STAT3, and actin levels serve as loading controls. Note the increased activating phosphorylation of FGFR3, STAT1, and STAT3 (arrow) in two independent FGFR3-M528I transfections, compared with two wt FGFR3 transfections. (C) NIH3T3 cells were transfected as indicated and analyzed for ERK activation by WB (pERK). pERK signal was quantified by densitometry and graphed (IOD). Total ERK and actin levels serve as loading controls, total FGFR3 levels serve as control for transfection. Note the levels of ERK phosphorylation which are increased in FGFR3-G380R and FGFR3-M528I when compared with wt FGFR3. (D) Reproducibility of increased ERK activation by FGFR3-M528I in three RCS transfections compared with three independent transfections of wt FGFR3. transmitted in an autosomal-dominant fashion. Our report also shows that functional studies are needed to confirm the pathogenicity of any novel or rare variant in this and other genes.

Recent functional studies for the ACH (OMIM 100800) mutations p.G380R and p.G375C have implied that these mutations increase the phosphorylation efficiency within unliganded FGFR3 dimers rather than increasing the crosslinking propensity of FGFR3 (14), although previous studies demonstrated that FGF ligand binding is essential for activation of mutated $F G F R 3(15,16)$. It is assumed that the FGFR3 mutations found in $\mathrm{HCH}$ also may result in constitutive activation of the receptor tyrosine kinase, but less than mutations causing $\mathrm{ACH}$ or thanatophoric dysplasia (3). The p.M528I mutation is located in the highly conserved intracellular TKD (conserved up to Tetraodon, see Fig. 3), just 12 amino acids away from the most common HCH mutation (p.N540K). The predictions according to software programs are similar to the predictions for the p.N540K mutation. We therefore anticipated the mutation p.M528I to be pathogenic and responsible for the observed phenotype. In order to functionally validate this prediction, we expressed FGFR3-M528I in RCS chondrocytes, which have successfully been used before to compare the relative levels of activation of different FGFR3 mutants associated with skeletal dysplasia (17). Figure 4A shows that FGFR3-M528I triggered ERK/MAP kinase activation in RCS cells to the levels similar to those induced by FGFR3-G380R, which is the most common mutation associated with $\mathrm{ACH}$. Similarly increased activation of ERK, STAT1, or STAT3 was found in two other cell models transfected with FGFR3-M528I, e.g. 293T cells and NIH3T3 cells (Fig. 4A and B). In $293 \mathrm{~T}$ cells, we also found an increased autophosphorylation of FGFR3-M528I when compared with wt FGFR3 (Fig. 4B). Collectively, these data confirm that M528I is indeed an activating mutation.

As mentioned earlier, M528 is situated in the cytosolic TKD. While the mutated site (M528) is outside all major active elements of the TKD, including the ATP binding site, activation loop, and/or substrate-binding site, our bioinformatics analysis suggests that it is located at the asymmetrical dimerization interface between two TKDs (Fig. 5). Formation of the asymmetrical TKD dimer appears essential for activation of the receptor tyrosine kinases from the auto-inhibited state (18). The activation of the TKD via asymmetrical dimerization presumes interaction of the C-lobe of the monomer $\mathrm{B}$ with the N-lobe of the TKD to be activated (monomer A) (Fig. 5) (18). Formation of the dimerization interface involving a conserved $\alpha$-helix 
A

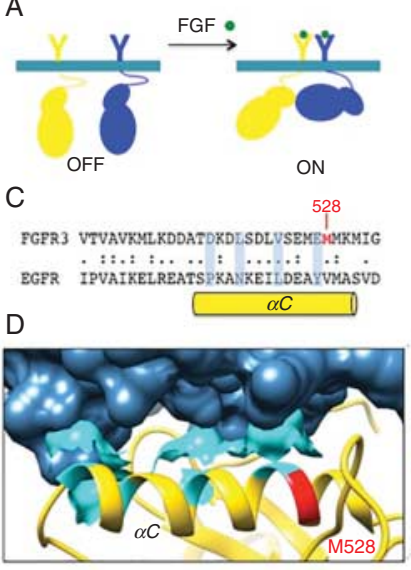

B

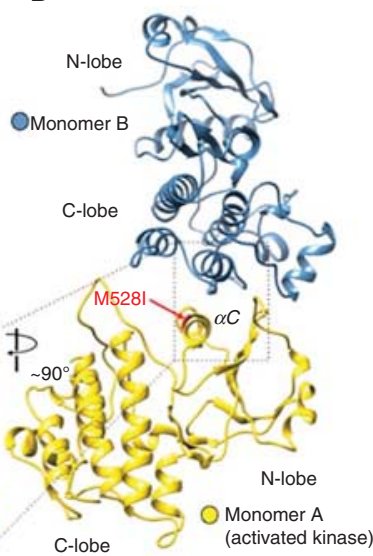

Figure 5

(A) A general model of the dimerization-dependent activation of FGFR3. (B) Homology model of the asymmetric dimer of the cytosolic TKD of FGFR3 based on the crystal structure of the kinase domain dimer of EGFR (PDB ID: 2GS6) (10). The model highlights the dimerization interface (dotted line) essential for activation of the kinase. The activating mutation, M528I, is located in the conserved $\alpha \mathrm{C}$ helix of the $\mathrm{N}$-terminal lobe of the activated kinase. The $\alpha \mathrm{C}$ helix is essential for formation of the dimerization interface and it participates in transduction of the allosteric signal from the dimerization interface to the kinase active site (10). (C) Local sequence alignment of the FGFR3 and the EGFR. Residues directly involved in formation of the dimerization interface as deduced from 2GS6 structure are displayed in the grey box. (D) Magnified view of the dimerization interface. The M528 adjoins a residue that is directly involved in the dimer formation. A full colour version of this figure is available at http://dx.doi.org/10.1530/EJE-14-0945.

( $\alpha C$ segment in Fig. 5) imposes allosteric modulation of the active site located between the $\mathrm{N}$ - and C-lobes of the TKD (18). Three alternative explanations for the activating phenotype of the M528I mutation can be deduced from the constructed model of the TKD dimer of the FGFR3 (Fig. 5). Firstly, the mutated residue might amplify allosteric signaling upon dimerization. Secondly, the mutated residue itself might be responsible for dissemination of the allosteric signal independent of the dimerization event. Thirdly, the M528I might promote TKD activation by stabilizing the interaction interface and promoting formation of the dimer. However, the latter explanation appears unlikely as the M528 residue is not directly involved in the dimerization interface, and the methionine and isoleucine are both hydrophobic amino acids with hydrophobicity values in the same range.

In addition to the most common p.N540K $\mathrm{HCH}$ mutation, other mutations have been reported to be associated with a milder phenotype with less severe disproportion, no macrocephaly, and minor radiologic abnormalities $(19,20,21)$. Mild forms have been described, especially where specific mutations in the p.K650 codon are involved (22). In patients with these mutations, growth retardation is less severe and the lumbar interpedicular distances and fibula/tibia length ratios are closer to normal, although disproportion of the upper extremities is as severe as in patients with other $\mathrm{HCH}$ mutations. A mild phenotype has also been described in families with a p.N540S mutation $(23,24,25)$. Patients with this mutation may have a height overlapping with the lower end of the normal range, but they all have radiologic abnormalities and some have a large HC.

The affected members of family A display an even milder phenotype, having short stature, a normal HC for age (though at the upper limit of the normal range if corrected for height SDS) (26), no disproportion, and a lack of radiologic signs of $\mathrm{HCH}$. All affected family members had $\mathrm{SH}: \mathrm{H}$ ratios around +1 SDS, which is to be expected for individuals with a height of approximately $-3 \operatorname{SDS}(8)$.

Family B shows an autosomal-dominant pattern of inheritance for short stature without disproportion and with a normal HC. Based on our finding in family A, we sequenced FGFR3 and found the rare variant c.1150T $>C$ (p.F384L) in the index patient, his sister and mother, who all shared the same growth pattern. However, further investigation in other family members (grandmother and maternal aunt) revealed that the mutation did not segregate with the short stature, which was in accordance with the prediction of a benign variant from several in silico prediction programs and the lack of altered ERK/MAP kinase activation in transfected RCS cells (data not shown). We therefore confirm that the FGFR3 variant in family $B$ is not pathogenic and another autosomal dominant cause of their short stature is suspected.

Our report contributes to the further delineation of the phenotype associated with FGFR3 mutations. The reported mutation in family $A$ is apparently linked to a phenotype of isolated short stature, whereas all other pathogenic mutations reported in FGFR3 cause disproportion, relative macrocephaly, and/or radiologic abnormalities besides the growth retardation. We suggest considering sequence analysis of the FGFR3 gene in patients with short stature, especially when there is an autosomal dominant pattern of inheritance, regardless of 
the body proportions. However, functional studies and proper segregation studies should be performed before concluding that the mutation involved is pathogenic.

\section{Supplementary data}

This is linked to the online version of the paper at http://dx.doi.org/10.1530/ EJE-14-0945.

\section{Declaration of interest}

The authors declare that there is no conflict of interest that could be perceived as prejudicing the impartiality of the research reported.

\section{Funding}

W Oostdijk received grant support from Novo Nordisk. P Krejci is supported by Ministry of Education, Youth and Sports of the Czech Republic (KONTAKT LH12004), and Grant Agency of Masaryk University (00712013). L Trantirek is supported by the project CEITEC (Central European Institute of Technology) (CZ.1.05/1.1.00/02.0068) from European Regional Development Fund.

\section{References}

1 Foldynova-Trantirkova S, Wilcox WR \& Krejci P. Sixteen years and counting: the current understanding of fibroblast growth factor receptor 3 (FGFR3) signaling in skeletal dysplasias. Human Mutation 201233 29-41. (doi:10.1002/humu.21636)

2 Cohen MM Jr. Some chondrodysplasias with short limbs: molecular perspectives. American Journal of Medical Genetics 2002112 304-313. (doi:10.1002/ajmg.10780)

3 Bober MB, Bellus GA, Nikkel SM \& Tiller GE. Hypochondroplasia. 1999 Jul 15 [Updated 2013 Sep 26]. In: Pagon RA, Adam MP, Ardinger HH, et al., editors. GeneReviews ${ }^{\circledR}$ [Internet]. Seattle (WA): University of Washington, Seattle; 1993-2015. Available from: http://www.ncbi. nlm.nih.gov/books/NBK1477/

4 Niklasson A, Ericson A, Fryer JG, Karlberg J, Larwence C \& Karlberg P. An update of the Swedish reference standards for weight, length and head circumeference at birth for given gestational age (1977-1981). Acta Paediatrica Scandinavica 199180 756-762. (doi:10.1111/j.16512227.1991.tb11945.x)

5 Schonbeck Y, Talma H, van DP, Bakker B, Buitendijk SE, Hirasing RA \& Van Buuren S. The world's tallest nation has stopped growing taller: the height of Dutch children from 1955 to 2009. Pediatric Research 201373 371-377. (doi:10.1038/pr.2012.189)

6 Cole TJ \& Roede MJ. Centiles of body mass index for Dutch children aged 0-20 years in 1980 - a baseline to assess recent trends in obesity. Annals of Human Biology 199926 303-308. (doi:10.1080/ 030144699282633)

7 Fredriks AM, Van Buuren S, Burgmeijer RJ, Meulmeester JF, Beuker RJ, Brugman E, Roede MJ, Verloove-Vanhorick SP \& Wit JM. Continuing positive secular growth change in The Netherlands 1955-1997. Pediatric Research 200047 316-323. (doi:10.1203/00006450-200003000-00006)

8 Fredriks AM, van Buuren S, van Heel WJ, Dijkman-Neerincx RH, Verloove-Vanhorick SP \& Wit JM. Nationwide age references for sitting height, leg length, and sitting height:height ratio, and their diagnostic value for disproportionate growth disorders. Archives of Disease in Childhood 200590 807-812. (doi:10.1136/adc.2004.050799)
9 Santen GW, Aten E, Sun Y, Almomani R, Gilissen C, Nielsen M, Kant SG, Snoeck IN, Peeters EA, Hilhorst-Hofstee Y et al. Mutations in SWI/SNF chromatin remodeling complex gene ARID1B cause Coffin-Siris syndrome. Nature Genetics 201244 379-380. (doi:10.1038/ ng.2217)

10 Kelley LA \& Sternberg MJ. Protein structure prediction on the web: a case study using the Phyre server. Nature Protocols 20094 363-371. (doi:10.1038/nprot.2009.2)

11 Pettersen EF, Goddard TD, Huang CC, Couch GS, Greenblatt DM, Meng EC \& Ferrin TE. UCSF Chimera - a visualization system for exploratory research and analysis. Journal of Computational Chemistry 200425 1605-1612. (doi:10.1002/jcc.20084)

12 Trujillo-Tiebas MJ, Riveiro R, Queipo A, Vallespin D, Cantalapiedra D, Lorda-Sanchez I \& Ayuso C. Gene symbol: FGFR3. Disease: skeletal dysplasia. Human Genetics 2004115 347-356. (doi:10.1007/s00439004-1161-3)

13 Bodian DL, McCutcheon JN, Kothiyal P, Huddleston KC, Iyer RK, Vockley JG \& Niederhuber JE. Germline variation in cancer-susceptibility genes in a healthy, ancestrally diverse cohort: implications for individual genome sequencing. PLoS ONE 20149 e94554. (doi:10.1371/ journal.pone.0094554)

14 He L, Serrano C, Niphadkar N, Shobnam N \& Hristova K. Effect of the G375C and G346E achondroplasia mutations on FGFR3 activation. PLOS ONE 20127 e34808. (doi:10.1371/journal.pone.0034808)

15 Monsonego-Ornan E, Adar R, Feferman T, Segev O \& Yayon A. The transmembrane mutation G380R in fibroblast growth factor receptor 3 uncouples ligand-mediated receptor activation from down-regulation. Molecular and Cellular Biology 200020 516-522. (doi:10.1128/MCB.20. 2.516-522.2000)

16 Iwata T, Li CL, Deng CX \& Francomano CA. Highly activated Fgfr3 with the $\mathrm{K} 644 \mathrm{M}$ mutation causes prolonged survival in severe dwarf mice. Human Molecular Genetics 200110 1255-1264. (doi:10.1093/hmg/10. 12.1255)

17 Krejci P, Salazar L, Kashiwada TA, Chlebova K, Salasova A, Thompson LM, Bryja V, Kozubik A \& Wilcox WR. Analysis of STAT1 activation by six FGFR3 mutants associated with skeletal dysplasia undermines dominant role of STAT1 in FGFR3 signaling in cartilage. PLOS ONE 20083 e3961. (doi:10.1371/journal.pone.0003961)

18 Zhang X, Gureasko J, Shen K, Cole PA \& Kuriyan J. An allosteric mechanism for activation of the kinase domain of epidermal growth factor receptor. Cell 2006125 1137-1149. (doi:10.1016/j.cell. 2006.05.013)

19 Rousseau F, Bonaventure J, Legeai-Mallet L, Schmidt H, Weissenbach J, Maroteaux P, Munnich A \& Le Merrer M. Clinical and genetic heterogeneity of hypochondroplasia. Journal of Medical Genetics 1996 33 749-752. (doi:10.1136/jmg.33.9.749)

20 Prinster C, Carrera P, Del Maschio M, Weber G, Maghnie M, Vigone MC, Mora S, Tonini G, Rigon F, Beluffi G et al. Comparison of clinical-radiological and molecular findings in hypochondroplasia. American Journal of Medical Genetics 199875 109-112. (doi:10.1002/ (SICI) 1096-8628(19980106)75:1 < 109::AID-AJMG22 > 3.0.CO;2-P)

21 Ramaswami U, Rumsby G, Hindmarsh PC \& Brook CG. Genotype and phenotype in hypochondroplasia. Journal of Pediatrics 1998133 99-102. (doi:10.1016/S0022-3476(98)70186-6)

22 Bellus GA, Spector EB, Speiser PW, Weaver CA, Garber AT, Bryke CR, Israel J, Rosengren SS, Webster MK, Donoghue DJ et al. Distinct missense mutations of the FGFR3 lys650 codon modulate receptor kinase activation and the severity of the skeletal dysplasia phenotype. American Journal of Human Genetics 200067 1411-1421. (doi:10.1086/316892)

23 Mortier G, Nuytinck L, Craen M, Renard JP, Leroy JG \& de Paepe A. Clinical and radiographic features of a family with hypochondroplasia owing to a novel Asn540Ser mutation in the fibroblast growth factor receptor 3 gene. Journal of Medical Genetics 200037 220-224. (doi:10.1136/jmg.37.3.220)

24 Thauvin-Robinet C, Faivre L, Lewin P, De Monléon JV, François C, Huet F, Couailler JF, Campos-Xavier AB, Bonaventure J \& Le Merrer M. 
Hypochondroplasia and stature within normal limits: another family with an Asn540Ser mutation in the fibroblast growth factor receptor 3 gene. American Journal of Medical Genetics 2003 119A 81-84. (doi:10.1002/ajmg.a.10238)

25 De Sanctis V, Baldi M, Marsciani A, Ravaioli E, Timoncini G, Reggiani L, Sensi A \& Zucchini A. ASN540SER mutation is associated with a mild form of hypochondroplasia: a 7 years follow-up in an Italian boy. Georgian Medical News 2012210 77-82.

26 Geraedts EJ, van Dommelen P, Caliebe J, Visser R, Ranke MB, van Buuren S, Wit JM \& Oostdijk W. Association between head circumference and body size. Hormone Research in Paediatrics 201175 213-219. (doi:10.1159/000321192)

Received 4 November 2014

Revised version received 8 March 2015

Accepted 16 March 2015 